\title{
Current Status of the Pixel Phase I Upgrade in CMS: Barrel Module Production
}

\author{
Rachel BARTEK*† \\ National Taiwan University (TW) \\ E-mail: rachel.bartek@cern.ch
}

The silicon pixel detector is the innermost component of the CMS tracking system, providing high precision space point measurements of charged particle trajectories. Before 2018 the instantaneous luminosity of the LHC is expected to reach about $2 \times 10^{34} \mathrm{~cm}^{-2} \mathrm{~s}^{-1}$, which will significantly increase the number of interactions per bunch crossing. To maintain a high tracking efficiency, CMS has planned to replace the current pixel system during phase I by a new lightweight detector, equipped with an additional 4th layer in the barrel, and one additional forward/backward disk. The present status of barrel modules production will be presented, including preliminary results from tests on the first production pixel modules of the new pixel tracker.

XXVII International Symposium on Lepton Photon Interactions at High Energies 17-22 August 2015

Ljubljana, Slovenia

${ }^{*}$ Speaker.

${ }^{\dagger}$ on behalf of the CMS Collaboration 


\section{Introduction}

The silicon pixel detector is the innermost part of the CMS experiment [1]. This high granularity detector experiences the most ionizing radiation because it is closest to the interaction point. Due to radiation damage the pixel detector needs to be replaced half way through run II. The phase I [2], or first replacement of the pixel detector in CMS is slated to be installed in the CMS experiment during an extended year end technical stop 2016/2017. Instead of replacing the pixel detector with a replica of the current pixel detector [3] in CMS, we take advantage of advances in read-out chip (ROC) technology and upgrade the overall pixel detector.

The first major change for the phase I pixel detector is the material budget as shown in Figure 1. One can see that there are gains in the material budget across the entire upgraded pixel detector even though we added one additional layer in the barrel and for each endcap (Figure 2). These gains are possible because the electronics have been moved out of the active area of the detector, and because of a new $\mathrm{CO}_{2}$ cooling system.

To allow for the innermost barrel pixel layer to move in from $4.3 \mathrm{~cm}$ to $3.0 \mathrm{~cm}$, a new beam pipe needed to be installed. This is essential for secondary vertex reconstruction in the busy environment of the LHC. However moving the innermost layer closer to the interaction point increases the particle flux passing through this layer of the pixel detector. To cope with both an expected increase of instantaneous luminosity provided by the LHC and being located closer to the interaction point, additional buffers have been put on the upgrade pixel ROC.

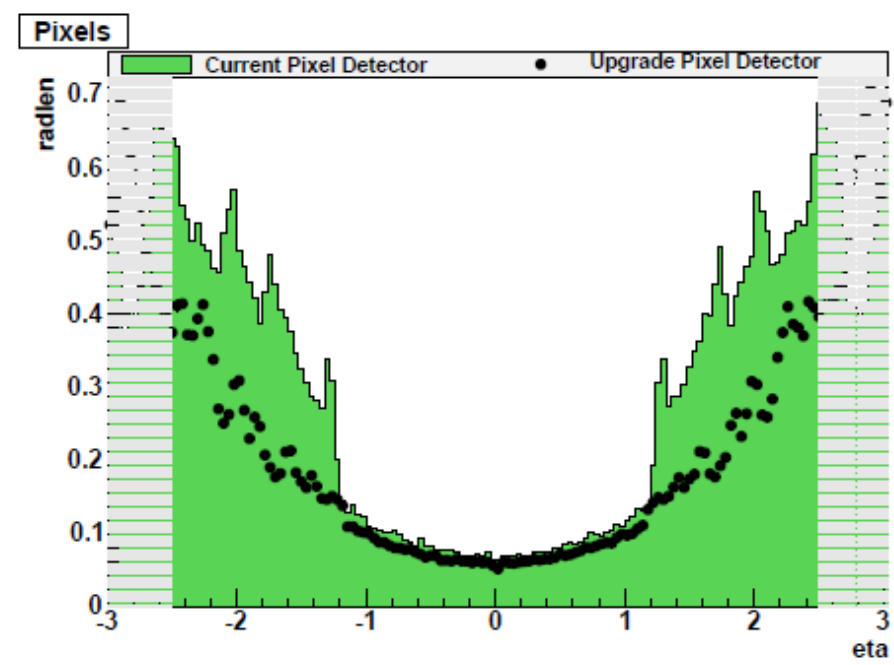

Figure 1: The material budget for the current pixel detector (solid green) and the upgrade pixel detector (black dots) in radiation lengths.

The phase I upgrade for the pixel detector in CMS is a large project, spanning new modules for the barrel and forward pixels, supply tube, support structure, cooling and front-end electronics. The number of modules needed for the four barrel layers, three inner disks, and three outer disks are listed in Table 1. This conference report will focus on barrel module production. 


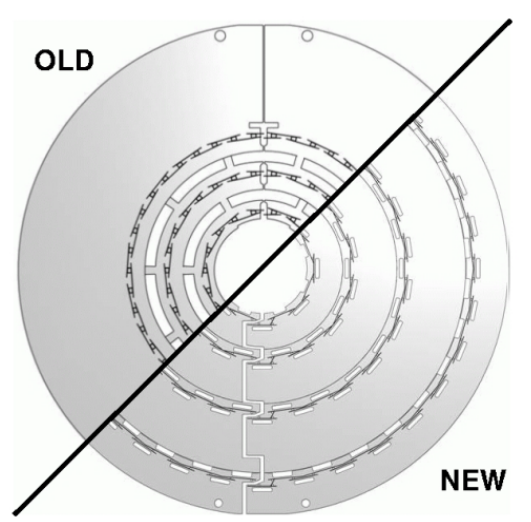

Figure 2: The location of the three layers of the current pixel detector (top left) with respect to the four layers of the pixel phase I upgrade (bottom right) are shown. The current pixel detector has layers located at radii: $4.3,7.2$, and $11 \mathrm{~cm}$. The phase I pixel detector will have layers located at radii: 3.0, 6.8, 10.9 and 16 $\mathrm{cm}$.

Table 1: The number of modules needed for the four barrel layers, three inner disks, and three outer disks.

\begin{tabular}{l|c}
\hline \hline Layer/Disk & Number of modules \\
\hline L1 & 96 \\
L2 & 224 \\
L3 & 352 \\
L4 & 512 \\
D1-3 inner & 264 \\
D1-3 outer & 408 \\
\hline \hline
\end{tabular}

\section{Barrel Module}

Barrel pixel modules consist of a twisted pair cable, high density interconnect (HDI), bare module, and base strips as shown in Figure 3. The twisted pair cable provides power and signal connections for the module. The HDI is a custom flex circuit. A bare module consists of silicon sensor with $\mathrm{n}^{+}$-in-n technology that can be placed under bias on top of 16 readout chips (ROCs) [4]. Each ROC reads out 26 double columns of 160 pixels each. The pixel pitch is $150 \mu \mathrm{m}$ x $100 \mu \mathrm{m}$. After the ROCs are bump-bonded to the sensor the module is called a bare module because it is missing the base strips and HDI.

Bare modules are tested before assembly. The pixels themselves and the bump bonds are tested. In Figure 4 a module with the highest quality grade is shown on top and a module with a bad double column is shown on bottom. In the pixel alive test 10 calibrate signals are sent to each pixel, if less than 10 readout signals are receives then pixel is considered 'dead'.

The barrel pixel modules for the phase I upgrade are constructed in several locations in Europe. The Swiss consortium, consisting of the PSI, ETH, and the University of Zurich, is building layers 1 and 2. The Italian consortium, consisting of INFN Bari, Catania, Firenze, Padova, and Pisa, is building half of layer 3. The other half of layer 3 is being built by CERN, NTU, and Helsinki. The 


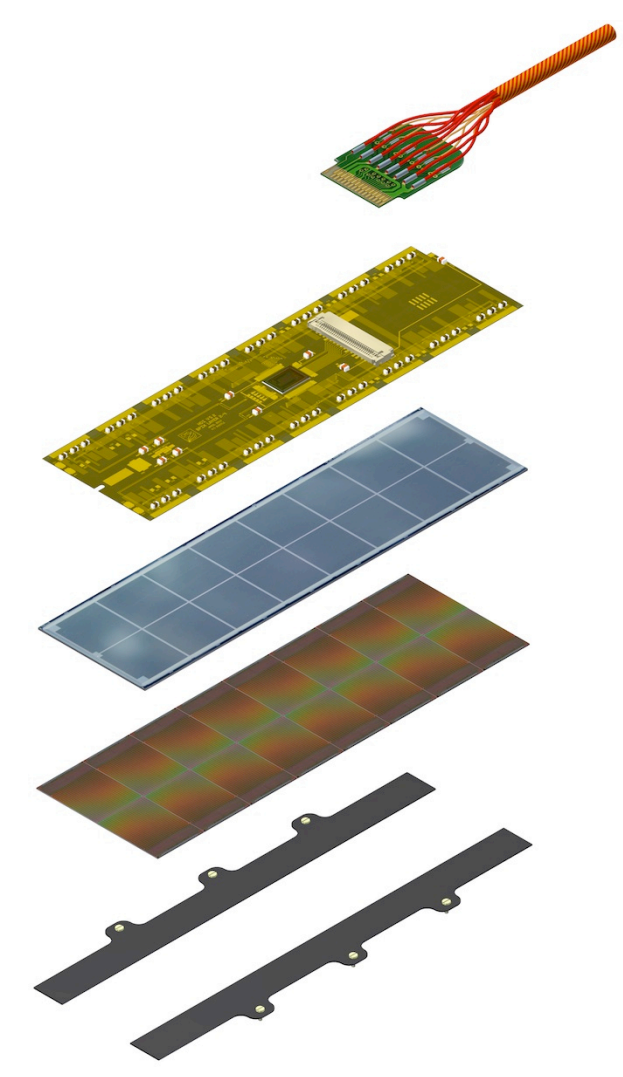

Figure 3: Exploded view of a barrel pixel module showing the twisted pair cable, HDI, silicon sensor, ROCs and base strips, from top to bottom.

German consortium, consisting of KIT, DESY, RWTH Aachen, and Hamburg University, is building layer 4. Bump bonds are placed on and then flip-chip bonded at Advacam in Finland, Dectris in Switzerland, IZM for the Italians, and DESY in Germany. For half of layer 4, KIT decided to have the American company RTI place the bumps, but does the flip-chip bonding, including the reflow, in house.

\section{HDI}

The appearance of a barrel pixel module is dominated by the gold HDI that covers most of its surface as seen in Figure 5. It is the interconnect between the token bit manager (TBM) [5] and the ROCs via 35 signal and power bonding pads located on the edge of the HDI for each ROC. As the name sugests, the TBM manages the token, or controls the sequence in which the pixels are read out. The TBM is a chip that sits at the center of the HDI.

The design of the HDI is optimized to be as thin as possible in units of interaction lengths. The HDI is $50 \mu \mathrm{m}$ thin. The thickest components mounted on the HDI are the Molex connector of $1.15 \mathrm{~mm}$ and the TBM of $100 \mu \mathrm{m}$. The thickest features can be clearly seen in the high rate X-ray test discussed in Section 5.2. 

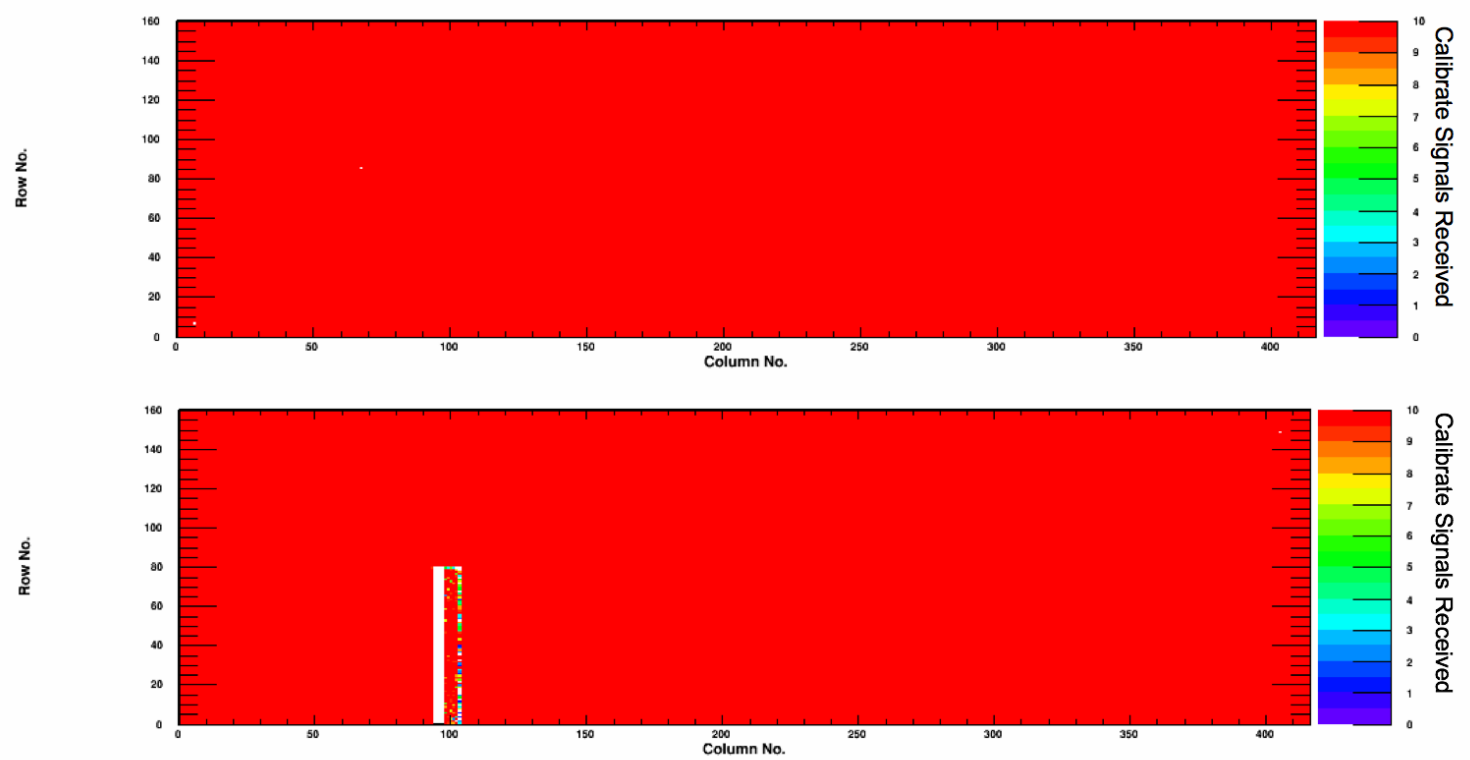

Figure 4: Results of the pixel alive test of a bare modules is shown for a module with the highest quality grade (top) and a module with a bad double column (bottom).

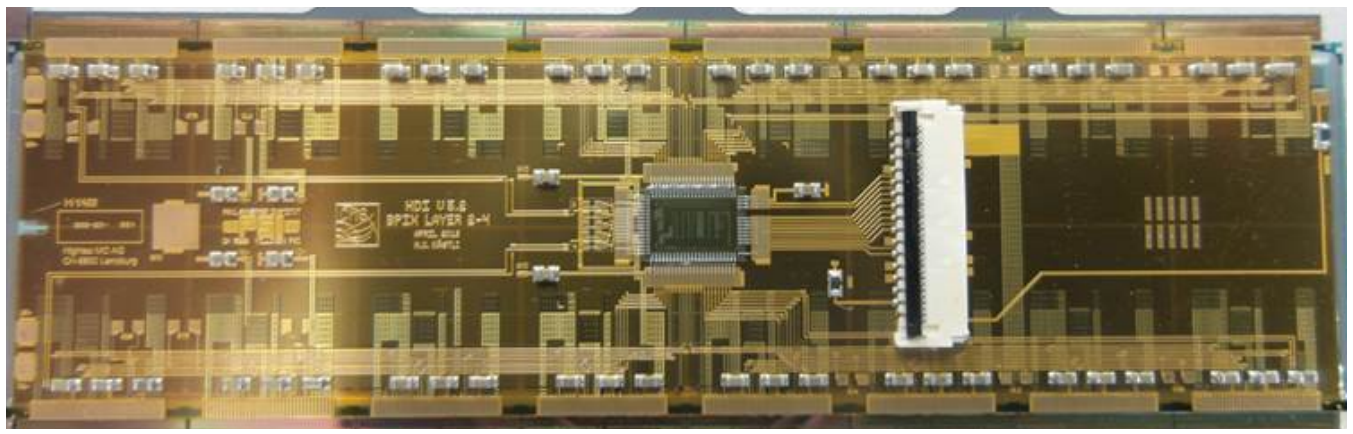

Figure 5: A picture of a production HDI with the TBM in its center. The white component is the Molex connector. Three sets of square pads in each quadrant are used for the electrical test.

There are several spy pads on the HDI. Three sets of square pads in each quadrant are used for the HDI electrical test. The largest pad on the HDI provides the ground for the electrical test. In addition to testing high voltage and low voltage supplied via the HDI, the electrical test checks for clock, calibration, trigger, reset, and serial data signals. Both polarities of each differential signal are read through a needle card test stand. An example test result is shown in Figure 6.

\section{Assembly}

Modules are assembled in two stages: base strip gluing and HDI gluing. First the base strips are glued under the bare module. Second the HDI is glued on top of the bare module. Identical jigs are used in five assembly centers across Europe. Both stages of gluing are done at CERN, Hamburg 


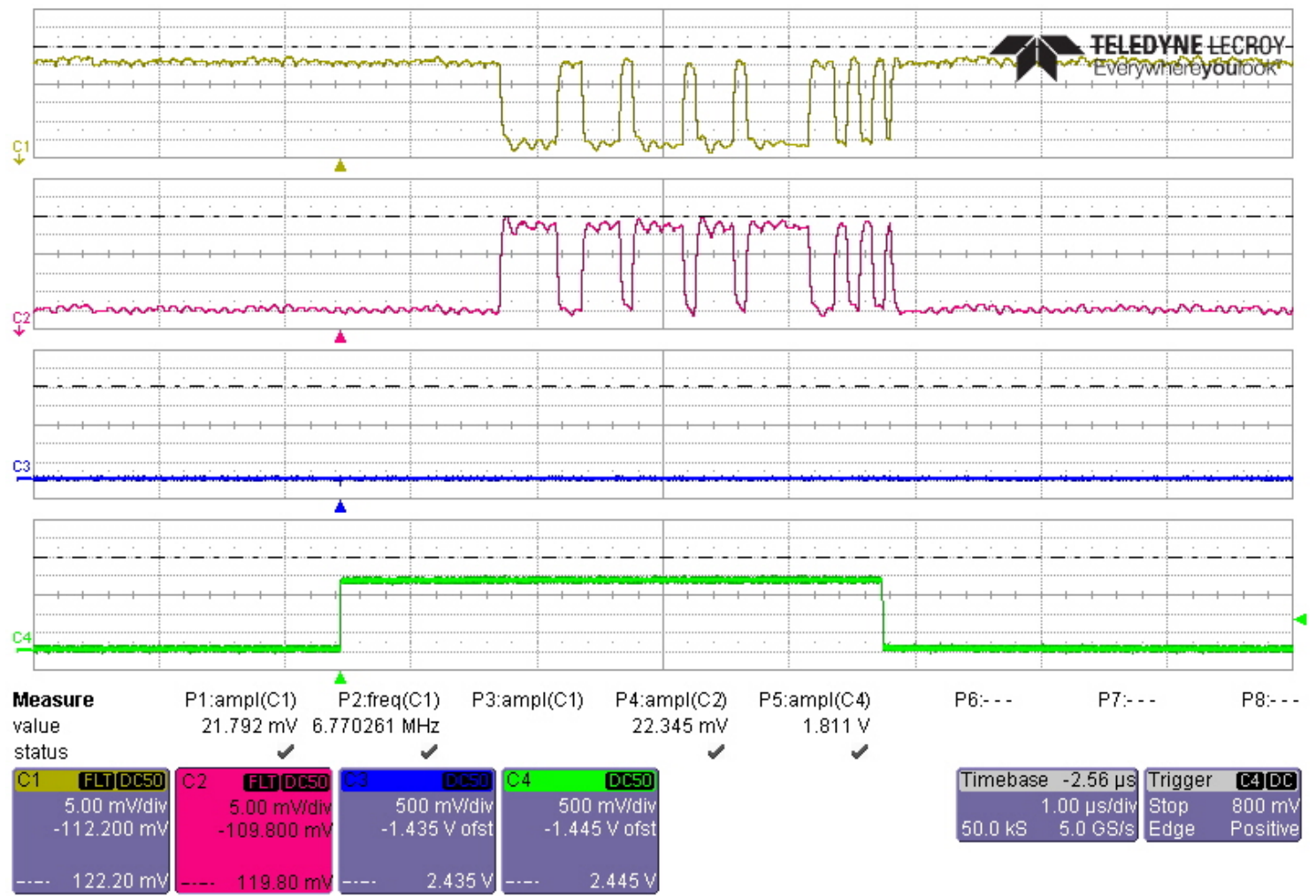

Figure 6: A screen shot from the oscilloscope is shown for the serial data test in one quadrant for an HDI. The yellow and pink lines are serial data of opposite polarities. The green signal indicated incoming data and serves as the trigger for this particular test.

University, PSI, and KIT. The Italian consortium chose to split assembly across two centers: Pisa glues the base strips on the bare module, then Bari glues the HDI on top. The glue used is Araldite 2011.

Alignment is a key issue for assembly. A precision of $50 \mu \mathrm{m}$ is required. A computer program was developed to use measurements from coordinate measurement machines to set micrometric screws on the jig for fine alignment.

A key challenge for base strip gluing is achieving full coverage of the base strips with glue, but not too much glue so that glue does not seep between the ROCs nor outside the base strip edges. The base strips serve as both the cooling element and the mechanical structure to hold the module in place. The glue conducts heat more efficiently than air, therefore an even layer of glue covering the entire surface between the base strip and the ROCs is essential for cooling the module. Tests with base strips and glass were performed to ensure good glue coverage. Some modules were glued to the jig after the base strip gluing step, indicating too much glue was used or the alignment was bad. After checking the alignment, the glue amount was adjusted by reducing the time the stamp which deposits the glue was dipped in the glue basin, and by redesigning the stamp.

The HDI provides bias voltage to the silicon sensor through several bonds from the bonding pad on the HDI to the sensor below. This is achieved due to a cut out on the upper right corner of the HDI shown in Figure 5. Gluing in this corner is crucial and challenging. One needs enough 
glue so that the HDI is well supported in this corner for bonding. However if there is too much glue, then the bonding pad on the sensor will be covered with glue and left unbondable.

\section{Full Qualification and Calibration}

After assembly modules are tested for functionality and then fully characterized in terms of performance. Only the most performant modules are selected to be used in the CMS detector.

\subsection{Cold Box Test}

A cold box is used to thermal cycle and test the modules for functionality and fully characterize the modules in terms of performance (pixel alive, S-curve, trimming, gain calibration). Modules undergo ten thermal cycles from $17^{\circ} \mathrm{C}$ to $-25^{\circ} \mathrm{C}$. The experience from construction of the previous CMS pixel detector was that $10 \%$ of modules failed during this thermal burn-in test [3]. Several modules were tested past 10 thermal cycles, but no degradation in performance was observed. This pixel detector is designed to run at $-10^{\circ} \mathrm{C}$.

After ten thermal cycles modules are tested for full functionality at $17^{\circ} \mathrm{C}$ and $-20^{\circ} \mathrm{C}$. More tests are performed than are in the scope of this conference report, but the details of a few tests are given below to give a sample of the level of validation required for pixel modules.

Every pixel is tested to measure its response to a given injected charge pulse. They are then trimmed, or tuned, to give a homogeneous response across the detector. An I-V curve is produced by changing the bias voltage in steps of 5 Volts from 0 to 500 Volts and measuring the corresponding leakage current. This measures the voltage where the leakage current breaks down. We require that the leakage current before break down is low for the best modules as the leakage current gets worse with radiation dose. All pixels are tested for bump bonding problems. The DAC threshold for each pixel is measured by injecting charge to a pad on the ROC surface, which creates a signal in the sensor, and by comparing this threshold to the one measured when charge is coupled to the pre-amplifier only via parasitic cross-talk. For the chip shown in Figure 7 (left), only a few bump bonds are bad and have a threshold difference of over 80 DAC units. Figure 7 (right) shows a ROC tested for noisy pixels by measuring the S-curve width. Pixels with over 500 electrons noise are bad and masked from the readout. These are just a few of the tests performed after thermal cycling.

\subsection{X-ray Tests}

An X-ray box is used to calibrate the modules and verify that they can withstand high rates. For the calibration test $\mathrm{X}$-rays are used to determine the number of electrons for a given pulseheight at a specific calibration voltage $\left(\mathrm{V}_{\text {cal }}\right)$ using fluorescence lines from four elements: zinc, silver, molybdenum, and tin. Each element has a know injection charge into the silicon sensor. A fit is performed to obtain the mean pulse-height per $\mathrm{V}_{\text {cal }}$ for each element. The pulse-height per $\mathrm{V}_{\text {cal }}$ for the four elements with a fit is shown in Figure 8 left with the resulting fit to the four elements on the right.

Pixel efficiencies at $50 \mathrm{MHz}$ and $120 \mathrm{MHz}$ are compared to ensure high pixel performance. In Figure 9 the shadow of the HDI can clearly be seen when the rates for each pixel from the high rate test are plotted.This test was still under development at the time of LP2015. 

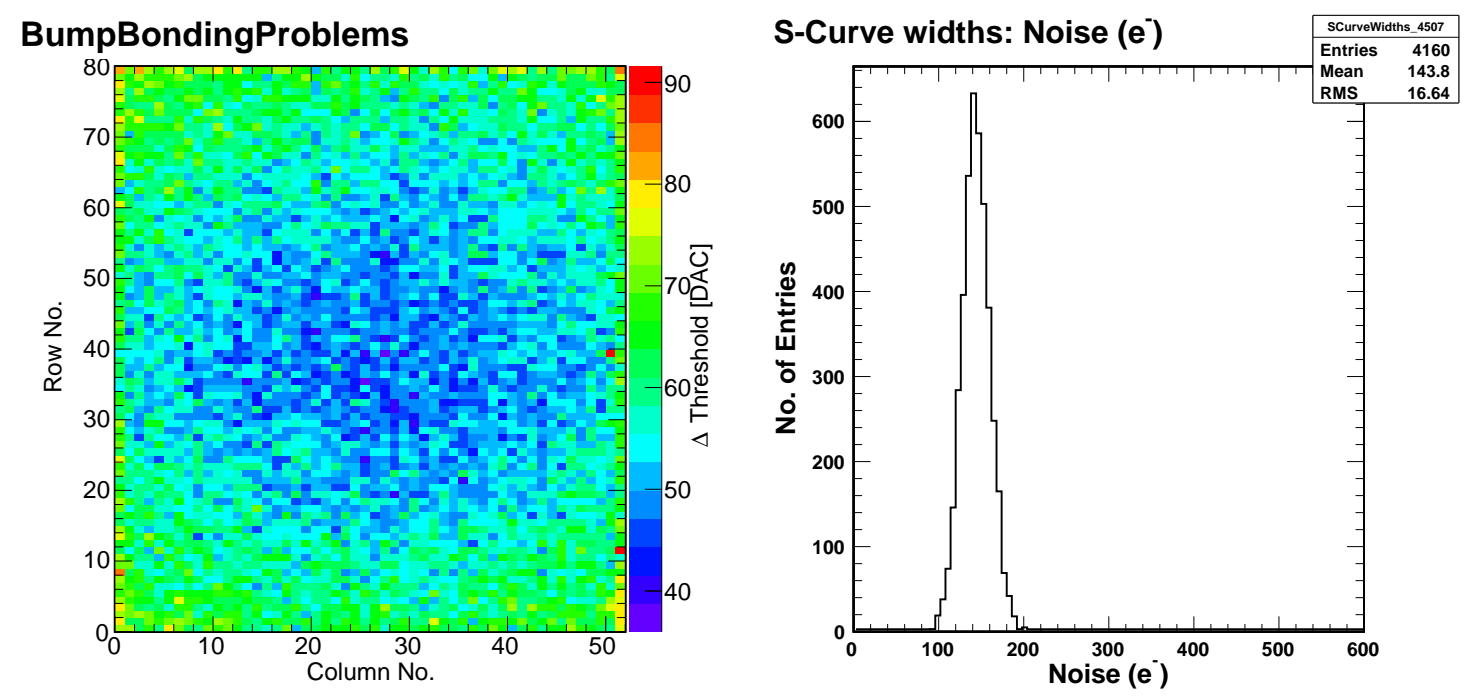

Figure 7: A ROC tested for bump bonding problems (left). Bump bonds with sensor and cross-talk DAC threshold differences over 80 DAC units are bad. A ROC tested for noisy pixels is shown on the right. Pixels with over 500 electrons noise are bad and masked from the readout.

\section{Summary}

This conference note summarizes the phase I pixel project for CMS, particularly, the start of production of barrel pixel modules. While production is underway some tests are still being finalized. Testing is done at each stage of assembly to ensure high quality pixel modules.

\section{References}

[1] CMS Collaboration, "The CMS experiment at the CERN LHC," JINST 3 (2008) S08004.

[2] CMS Collaboration, "CMS Technical Design Report for the Pixel Detector Upgrade," Tech. Rep. CERN-LHCC-2012-016. CMS-TDR-11, CERN, Geneva, Sep, 2012.

[3] CMS Collaboration, "The CMS tracker: addendum to the Technical Design Report," Tech. Rep. CERN-LHCC-2000-016. CMS-TDR-5-add-1, Geneva, 2000.

[4] B. Meier, "CMS pixel detector with new digital readout architecture," Journal of Instrumentation 6 no. 01, (2011) C01011.

[5] E. Bartz, "The token bit manager chip for the CMS pixel readout," in 9th Workshop on Electronics for LHC Experiments, pp. 185-189. Amsterdam, 2003. 

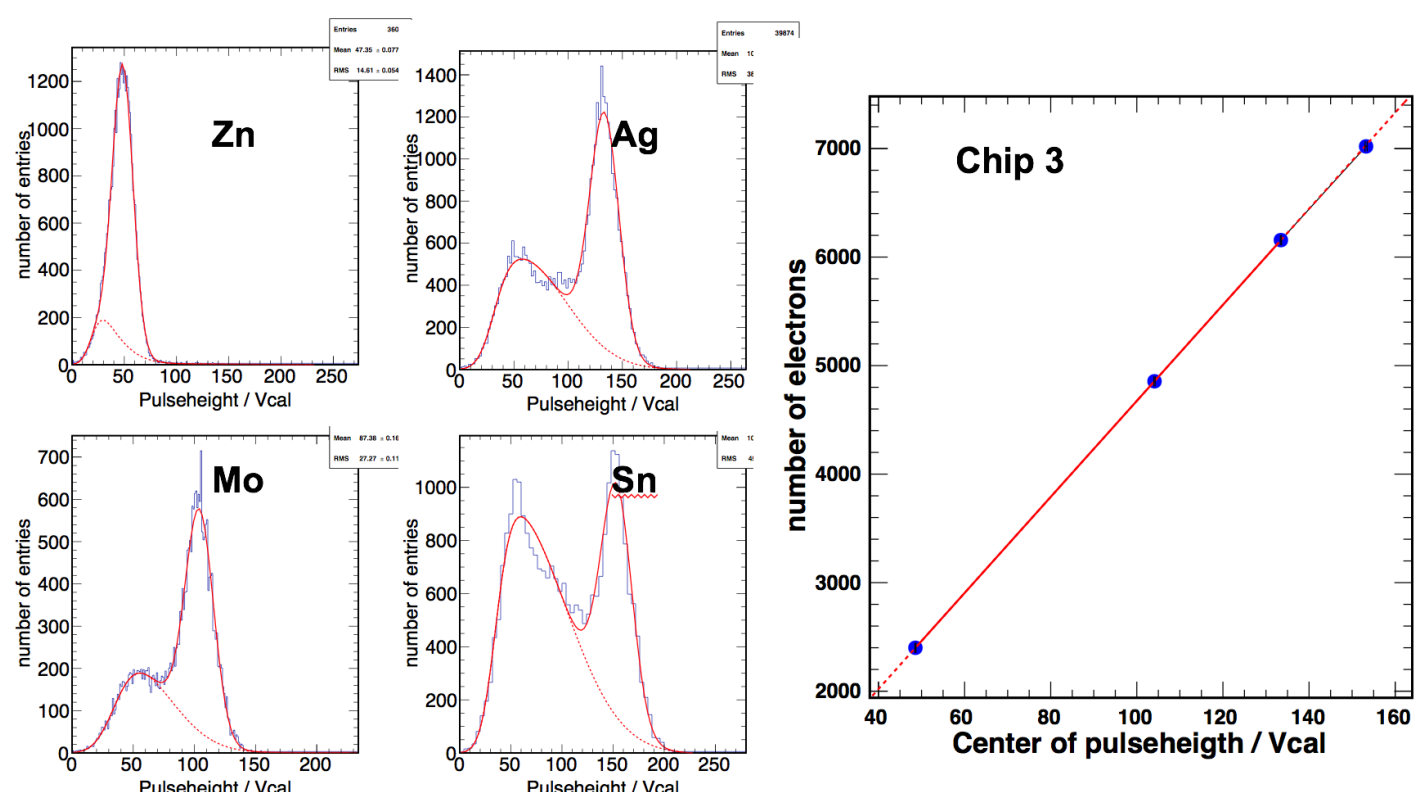

Figure 8: The calibration data for the four elements with a fit is shown (left) with the resulting fit to the four elements on the right.

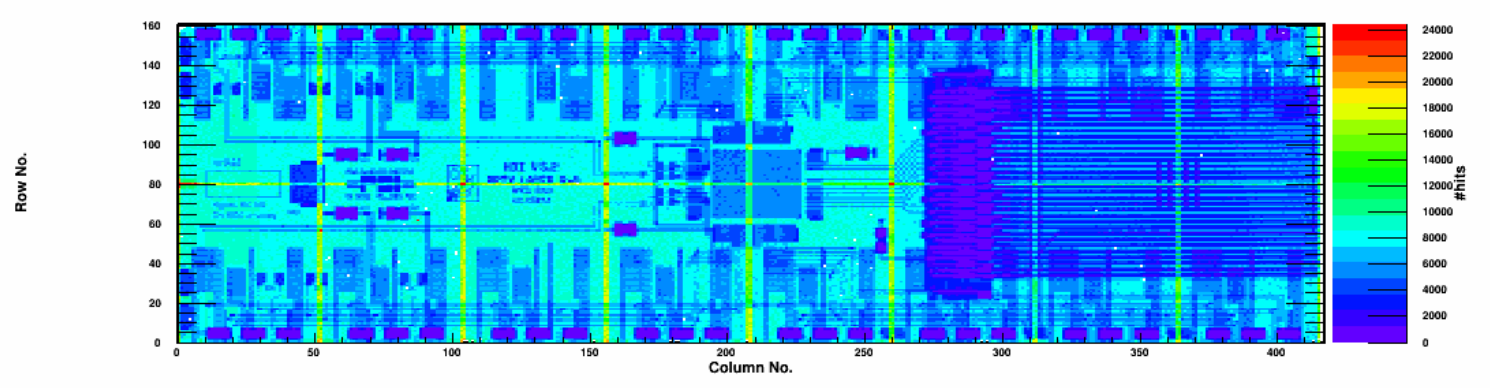

Figure 9: The shadow of the HDI can clearly be seen when the rates for each pixel from the high rate test are plotted. 